\title{
IncRNA LINC-PINT is downregulated in melanoma and regulates cell proliferation by downregulating IncRNA BANCR
}

\author{
QING HUANG ${ }^{1,2^{*}}$, DELI ZHANG ${ }^{3,4^{*}}$, QINGCHUN DIAO ${ }^{3}$ and MAO LIN ${ }^{3}$ \\ ${ }^{1}$ Department of Dermatology, Chongqing Shapingba District People's Hospital; ${ }^{2}$ Department of Dermatology, \\ The First Affiliated Hospital of Chongqing Medical University; ${ }^{3}$ Department of Dermatology, \\ Chongqing Traditional Chinese Medicine Hospital, Chongqing 400011; ${ }^{4}$ Department of Dermatology, \\ School of Clinical Medicine, Southwest Medical University, Luzhou, Sichuan 646000, P.R. China
}

Received December 24, 2018; Accepted June 13, 2019

DOI: $10.3892 / \mathrm{ol} .2019 .10631$

\begin{abstract}
The development of melanoma may involve long non-coding RNAs (lncRNAs); however, the functions of the majority of lncRNAs in melanoma are unknown. The present study investigated the role of long intergenic non-protein coding RNA p53 induced transcript (LINC-PINT) in melanoma. In the present study, quantitative PCR was used to detect gene expression, overexpression experiments were performed to analyze gene interactions and CCK- 8 assays were used to analyze cell proliferation. LINC-PINT was downregulated, while BRAF-activated non-coding RNA (BANCR) was upregulated in melanoma tissues compared with normal adjacent tissues. Expression levels of LINC-PINT decreased, while expression levels of BANCR increased with increasing tumor thickness. The expression levels of LINC-PINT and BANCR were inversely associated in melanoma tissues but not in healthy adjacent tissue. LINC-PINT overexpression downregulated BANCR expression in melanoma cells, while BANCR overexpression did not significantly affect LINC-PINT expression. LINC-PINT overexpression inhibited melanoma cell proliferation in vitro compared to controls. BANCR overexpression attenuated the effects of LINC-PINT overexpression. The present study revealed that lncRNA LINC-PINT is downregulated in melanoma and may regulate melanoma cell proliferation by downregulating lncRNA BANCR.
\end{abstract}

Correspondence to: Dr Mao Lin, Department of Dermatology, Chongqing Traditional Chinese Medicine Hospital, 40 Daomenkou Road, Chongqing 400011, P.R. China

E-mail: ha90434@163.com

${ }^{*}$ Contributed equally

Key words: melanoma, long non-coding RNA LINC-PINT, long non-coding BANCR, proliferation

\section{Introduction}

As the most aggressive skin cancer, melanoma is characterized by the rapid progression (1). Surgical resection of primary tumors usually results in satisfactory outcomes for patients at early stages (2). However, the development of melanoma is usually accompanied by tumor metastasis to regional lymph nodes or even distant organs, which lacks radical treatment (3). At present, the 5-year survival rate of metastatic melanoma patients remains $<20 \%$ (4). The unclear pathogenesis of melanoma is the major challenge for clinical treatment of this disease (5). Identification of novel therapeutic targets is always needed to improve the survival of melanoma patients.

Long non-coding RNAs (lncRNAs) are RNA transcripts consisting of $>200$ nucleotides with no protein-coding capacity (6). Different from messenger RNAs, lncRNAs participate in cellular processes by regulating gene expression at post-transcriptional and translational levels, or even through epigenetic pathways $(6,7)$. There is mounting evidence that lncRNAs are critical determinants in human diseases, and dysregulated lncRNA expression is closely correlated with the occurrence of many cancers (8). Regulation of IncRNA expression has been proven as potential therapeutic target for cancer treatment (9). However, function of most lncRNAs remains unclear. LncRNA LINC-PINT is a recently identified tumor suppressor in different types of cancer, such as lymphoblastic leukemia $(10,11)$. In the present study we investigate the involvement of LINC-PINT in melanoma, and explored its interactions with BANCR, which promotes melanoma (12).

\section{Materials and methods}

Research subjects. A total of 60 patients with melanoma [ 35 males and 25 females; age range, 28 to 69 years; mean age, 49.7 \pm 5.6 (standard deviation) years] were enrolled in Chongqing Traditional Chinese Medicine Hospital (Chongqing, China) between January 2015 and January 2018. All patients were diagnosed pathologically by at least 3 experienced pathologists. The inclusion criteria for enrolment in the current study were as follows: i) Patients with melanoma patients with no history of other malignancies; and ii) patients willing to 
participate. The exclusion criteria were as follows: i) Patients complicated with other skin diseases or other severe diseases, including other types of cancer; and ii) patients who had been treated within 3 months prior to admission. According to the American Joint Committee on Cancer staging (13), there were 23, 18 and 19 cases at stage I, II and III, respectively. According to the thickness of primary tumors, there were 15 cases $<1 \mathrm{~mm}, 16$ cases between $1-2 \mathrm{~mm}, 15$ cases between $2-4 \mathrm{~mm}$ and 14 cases $>4 \mathrm{~mm}$. The current study was approved by the Ethics Committee of Chongqing Traditional Chinese Medicine Hospital (Chongqing, China). All patients signed informed consent.

Specimens and cell lines. Tumor tissues and adjacent (within $2 \mathrm{~cm}$ around tumors) healthy tissues were collected through biopsy and were stored in a liquid nitrogen sink at $-196^{\circ} \mathrm{C}$ prior to use. Tissues were stored in liquid nitrogen prior to use. The melanoma cell lines A375-P and A375-MA2 (ATCC; American Type Culture Collection, Manassas, VA, USA) were used in the current study. Cells were cultured using ATCC-formulated Dulbecco's Modified Eagle Medium (American Type Culture Collection) containing 10\% fetal bovine serum (American Type Culture Collection) in an incubator at $37^{\circ} \mathrm{C}$ and $5 \% \mathrm{CO}_{2}$.

Total RNA extraction and reverse-transcription quantitative polymerase chain reaction $(R T-q P C R)$. $\mathrm{RNAzol}^{\circledR}$ reagent (GeneCopoeia, Inc., Rockville, MD, USA) was used to extract total RNA from tissue specimens and in vitro cultured A375-P and A375-MA2 cells. Tissues were ground in liquid nitrogen prior to the addition of RNAzol ${ }^{\circledR}$ reagent. A RevertAid RT Reverse Transcription Kit (Thermo Fisher Scientific, Inc., Waltham, MA, USA) was used to synthesize cDNA through reverse transcription using the following conditions: $25^{\circ} \mathrm{C}$ for $5 \mathrm{~min}, 55^{\circ} \mathrm{C}$ for $30 \mathrm{~min}$ and $80^{\circ} \mathrm{C}$ for $15 \mathrm{~min}$. To detect the expression of LINC-PINT and BANCR, SYBR ${ }^{\mathrm{TM}}-$ Green Master mix (Thermo Fisher Scientific, Inc.) was used to prepare all PCR reaction systems. CFX96 Touch ${ }^{\mathrm{TM}}$ Real-Time PCR Detection system (Bio-Rad Laboratories, Inc., Hercules, CA, USA) was used to perform all PCR reactions with $18 \mathrm{~S}$ RNA as endogenous control. Primer sequences were as follows: LINC-PINT, forward, 5'-CGTGGGAGCCCCTTT AAGTT-3' and reverse, 5'-GGGAGGTGGCGTAGTTTC TC-3'; BANCR forward, 5'-ACAGGACTCCATGGCAAA CG-3' and reverse, 5'-ATGAAGAAAGCCTGGTGCAGT-3'; and $18 \mathrm{~S}$ forward, 5'-GCTTAATTTGACTCAACACGGGA-3' and reverse, 5'-AGCTATCAATCTGTCAATCCTGTC-3'. The following thermocylcing conditions were used: $95^{\circ} \mathrm{C}$ for $30 \mathrm{sec}$, followed by 40 cycles of $95^{\circ} \mathrm{C}$ for $10 \mathrm{sec}$ and $58^{\circ} \mathrm{C}$ for $35 \mathrm{sec}$ and a final extension step at $72^{\circ} \mathrm{C}$ for $40 \mathrm{sec}$. Expression of LINC-PINT and BANCR was normalized to $18 \mathrm{~S}$ using the $2^{-\Delta \Delta \mathrm{Cq}}$ method (14).

Vectors and cell transfection. pcDNA3.1 vectors expressing LINC-PINT and BANCR were designed and constructed by Sangon Biotech Co., Ltd. (Shanghai, China). A375-P and A375-MA2 cells were cultured overnight to reach $70-80 \%$ confluence, followed by cell transfection performed using Lipofectamine ${ }^{\circledR} 3000$ (Thermo Fisher Scientific, Inc.) according to the manufacturer's protocol, with $10 \mathrm{nM}$ LINC-PINT and BANCR vectors or empty vectors (negative control, NC). Untransfected cells were were used as control (C) cells. Cells were harvested $24 \mathrm{~h}$ following transfection and used for subsequent experimentation. Transfection efficiency was determined using RT-qPCR.

Cell proliferation assay. The Cell Counting Kit-8 (CCK-8) (Beyotime Institute of Biotechnology, Haimen, China) was used to measure the cell proliferation rate $24 \mathrm{~h}$ following transfection. Cells were collected and single cell suspensions were prepared. Cell density was adjusted to $5 \times 10^{4}$ cells $/ \mathrm{ml}$. Each well of a 96-well plate was filled with $100 \mu \mathrm{l}$ cell suspension. The plate was incubated at $37^{\circ} \mathrm{C}$ in a $5 \% \mathrm{CO}_{2}$ incubator, followed by the addition of $10 \mu \mathrm{lCCK}-8$ solution $24,28,72$ and $96 \mathrm{~h}$ later. The cells were subsequently incubated for an additional $4 \mathrm{~h}$ at $37^{\circ} \mathrm{C}$. After adding $10 \mu \mathrm{l}$ DMSO, optical density values at a wavelength of $450 \mathrm{~nm}$ were measured to assess cell proliferation.

Statistical analysis. Three biological replicates were performed for each experiment. GraphPad Prism software (version 6; GraphPad Software, Inc., La Jolla, CA, USA) was used to process the data and perform statistical analysis. Data are expressed as the mean \pm standard deviation. Comparisons of expression levels of LINC-PINT and BANCR between melanoma and healthy adjacent tissues were performed using a paired t-test. Comparisons of LINC-PINT and BANCR among the tumor thickness groups, as well as comparisons of expression levels of LINC-PINT and BANCR and cell proliferation data among cell groups were performed by one-way ANOVA followed by a Tukey post hoc test. Associations between LINC-PINT and BANCR were analyzed by linear regression. $\mathrm{P}<0.05$ was considered to indicate a statistically significant difference.

\section{Results}

Expression of LINC-PINT and BANCR is altered in melanoma tissues. The expression levels of LINC-PINT and BANCR in 60 patients with melanoma were detected by RT-qPCR. Compared with healthy adjacent tissues, LINC-PINT was significantly downregulated in tumor tissues (Fig. 1A; $\mathrm{P}<0.05)$. By contrast, BANCR was significantly upregulated in tumor tissues compared with healthy adjacent tissues (Fig. 1B; $\mathrm{P}<0.05)$.

Expression of LINC-PINT and BANCR is affected by tumor thickness. Primary tumors were classified based on thickness. There were 15 cases $<1 \mathrm{~mm}, 16$ cases between $1-2 \mathrm{~mm}$, 15 cases between $2-4 \mathrm{~mm}$ and 14 cases $>4 \mathrm{~mm}$. The expression levels of LINC-PINT significantly decreased (Fig. 2A; $\mathrm{P}<0.05$ ), while the expression levels of BANCR significantly increased (Fig. 2B; $\mathrm{P}<0.05$ ) with increasing tumor thickness.

LINC-PINT andBANCR are inverselyassociated.Associations between LINC-PINT and BANCR were analyzed by linear regression. The expression levels of LINC-PINT and BANCR were significantly and inversely associated in melanoma tissues (Fig. 3A; P<0.01). However, LINC-PINT and BANCR expression levels were not significantly associated in healthy adjacent tissues (Fig. 3B; $\mathrm{P}=0.57$ ). 

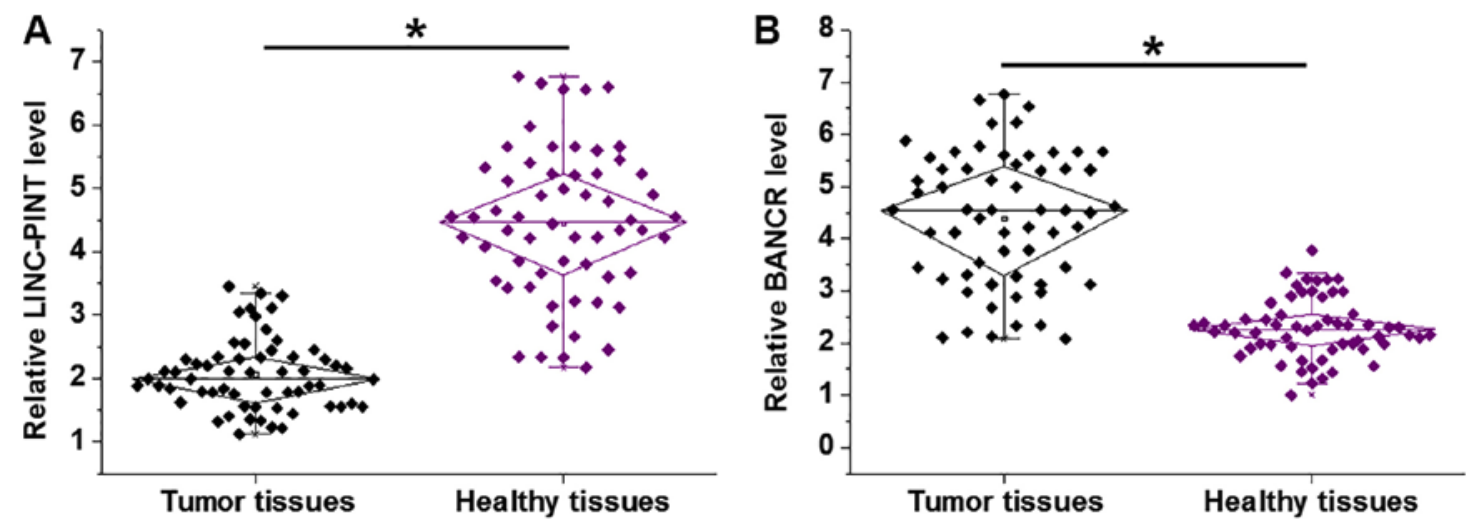

Figure 1. Expression levels of LINC-PINT and BANCR were altered in melanoma tissues. Reverse-transcription quantitative polymerase chain reaction results revealed that (A) LINC-PINT was downregulated while (B) BANCR was upregulated in tumor tissues compared with healthy adjacent tissues obtained from patients with melanoma. ${ }^{~} \mathrm{P}<0.05$, as indicated. LINC-PINT, long intergenic non-protein coding RNA p53 induced transcript; BANCR, BRAF-activated non-protein coding RNA.

A

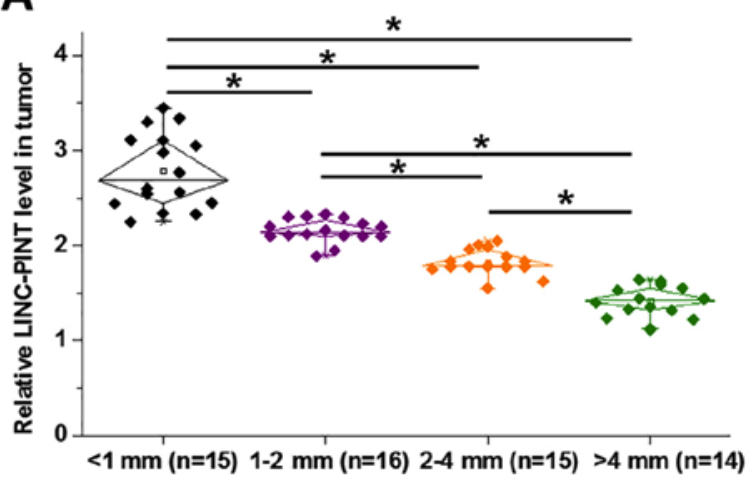

B

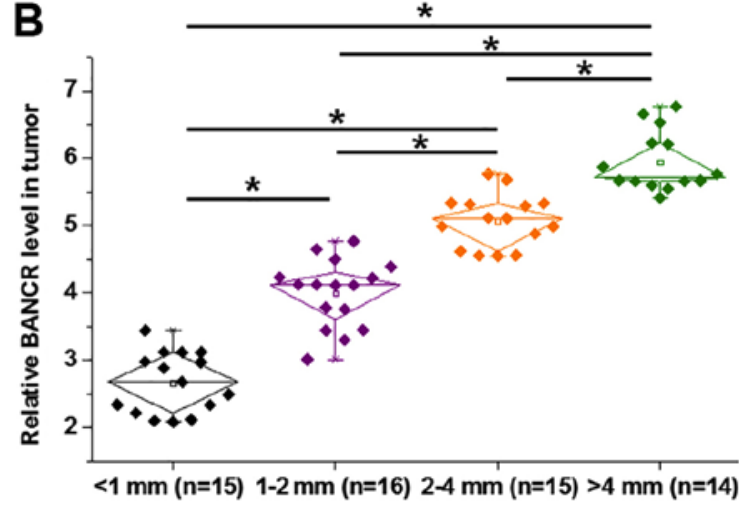

Figure 2. Expression of LINC-PINT and BANCR was affected by the thickness of tumors. Results of reverse-transcription quantitative polymerase chain reaction revealed that (A) the expression levels of LINC-PINT significantly decreased and (B) the expression levels of BANCR significantly increased with increasing tumor thickness. ${ }^{*} \mathrm{P}<0.05$, as indicated. LINC-PINT, long intergenic non-protein coding RNA p53 induced transcript; BANCR, BRAF-activated non-protein coding RNA.

LINC-PINT is a likely upstream inhibitor of BANCR in melanoma cells. The significantly inverse association between LINC-PINT and BANCR in tumor tissues indicated the possible interactions between LINC-PINT and BANCR. To further investigate the interaction between LINC-PINT and BANCR, vectors expressing LINC-PINT and BANCR were transfected into A375-P and A375-MA2 melanoma cell lines. Overexpression in A375-P and A375-MA2 cells was achieved $24 \mathrm{~h}$ following transfection and compared with untransfected cells (C group) and cells transfected with empty vectors (NC group; Fig. 4A; $\mathrm{P}<0.05)$. Compared with the $\mathrm{C}$ and $\mathrm{NC}$ groups, cells overexpressing LINC-PINT revealed significantly downregulated BANCR levels (Fig. 4B; P<0.05), while cells with BANCR overexpression revealed no significant changes in the LINC-PINT expression level compared with the C and NC groups (Fig. 4C; $\mathrm{P}>0.05$ ).

LINC-PINT overexpression inhibits melanoma cell proliferation through BANCR. LINC-PINT and BANCR expression levels were significant increased following co-transfection with LINC-PINT and BANCR expression vectors compared with the $\mathrm{C}$ and $\mathrm{NC}$ groups (Fig. 5A; $\mathrm{P}<0.05$ ). Compared with untransfected cells (C group) and cells transfected with empty vectors (NC group), cell proliferation was decreased in cells overexpressing LINC-PINT at $96 \mathrm{~h}$ (Fig. 5B; $\mathrm{P}<0.05$ ). BANCR overexpression increased proliferation compared with the $\mathrm{C}$ and $\mathrm{NC}$ groups (Fig. 5B; $\mathrm{P}<0.05$ ). Co-transfection with LINC-PINT and BANCR expression vectors attenuated the effects of LINC-PINT overexpression (Fig. 5B; $\mathrm{P}<0.05$ ).

\section{Discussion}

LINC-PINT is a recently identified tumor suppressor in different types of cancer, including retinoblastoma and gastric cancer $(10,11)$; however, its role in melanoma remains unknown. The current study, to the best of our knowledge, was the first to show the downregulated expression pattern of LINC-PINT in melanoma, and suggested that LINC-PINT may be a tumor suppressor in this disease. Furthermore, the current study demonstrated that the actions of LINC-PINT in melanoma are likely achieved through the interaction with BANCR.

BANCR is a well-characterized oncogenic lncRNA in different types of cancer, including retinoblastoma and gastric cancer $(12,15,16)$. Upregulation of BANCR promoted tumor 

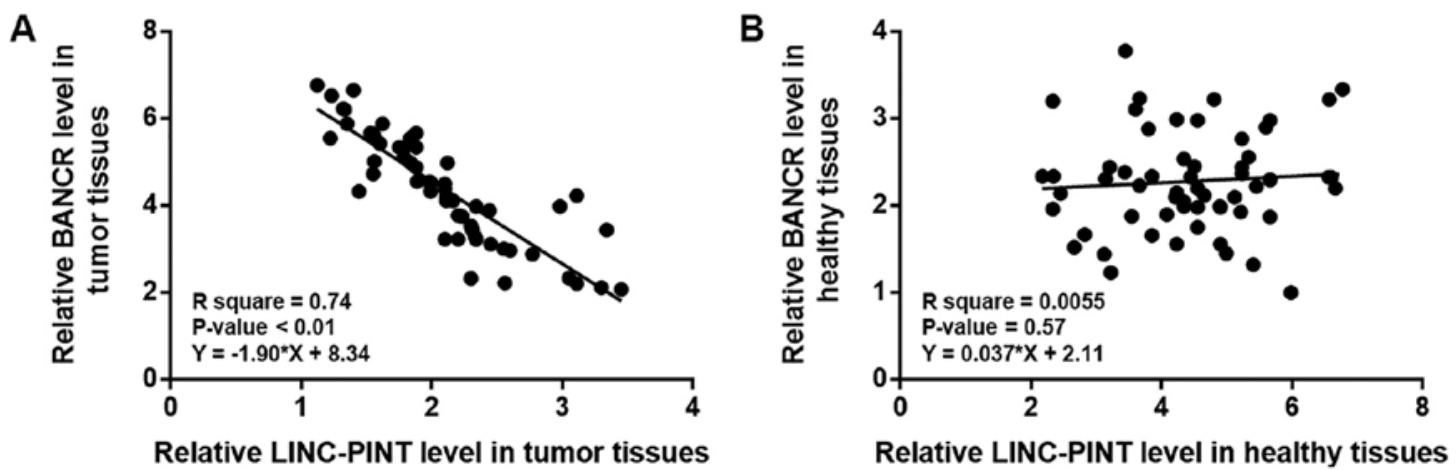

Figure 3. LINC-PINT and BANCR expression levels were inversely associated in tumor tissues. Linear regression analysis revealed that the expression levels of LINC-PINT and BANCR were significantly and inversely associated in (A) melanoma tissues but not in (B) healthy adjacent tissues. LINC-PINT, long intergenic non-protein coding RNA 53 induced transcript; BANCR, BRAF-activated non-protein coding RNA.
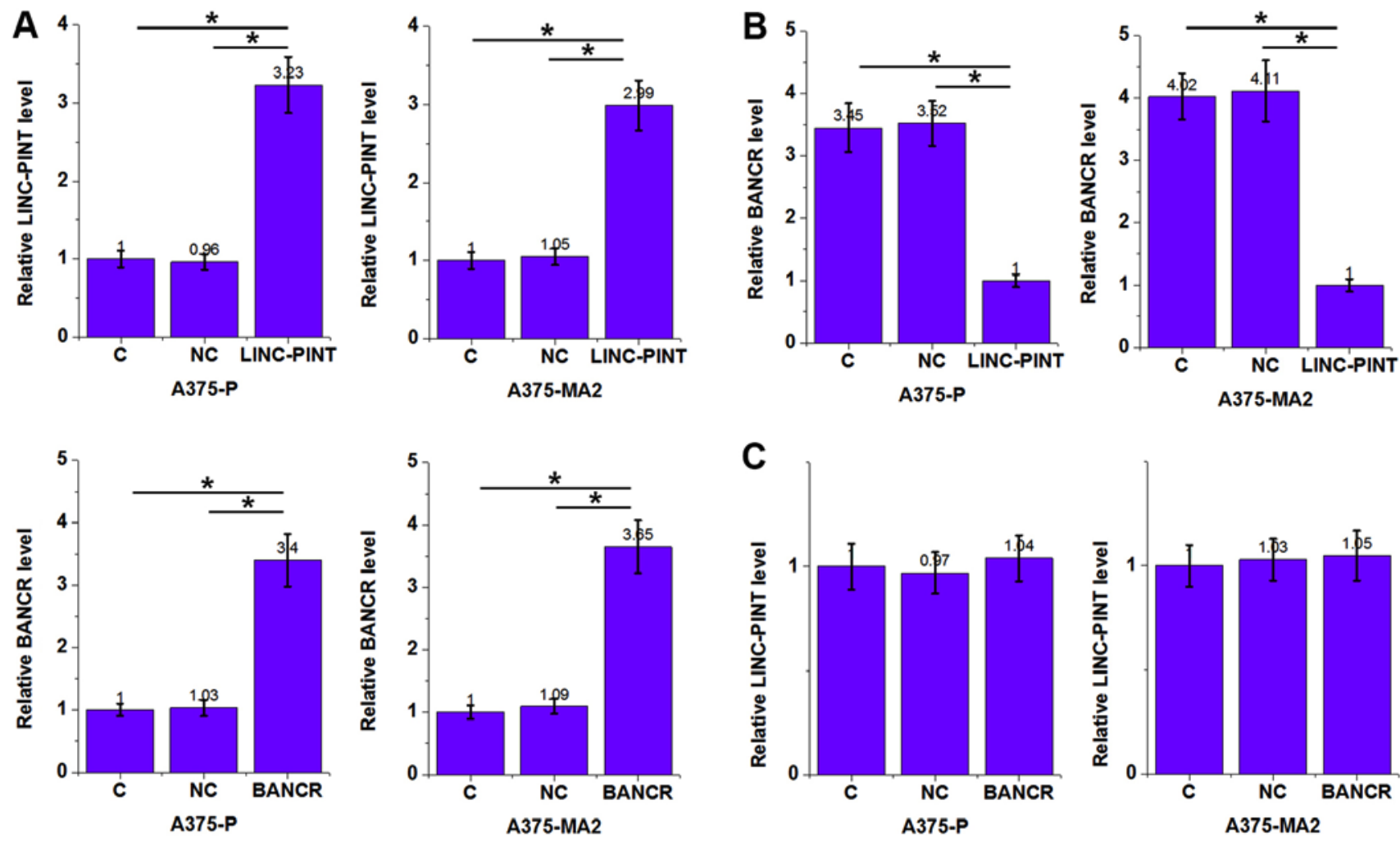

Figure 4. LINC-PINT is a likely upstream inhibitor of BANCR in melanoma cells. (A) Overexpression of LINC-PINT and BANCR in A375-P and A375-MA2 melanoma cells was achieved $24 \mathrm{~h}$ following transfection. (B) LINC-PINT overexpression resulted in BANCR downregulation in both cell lines. (C) BANCR overexpression did not significantly affect LINC-PINT expression. The numbers above each bar indicate the mean value of three replicates. "P $<0.05$, as indicated. LINC-PINT, long intergenic non-protein coding RNA p53 induced transcript; BANCR, BRAF-activated non-protein coding RNA; C, control (untransfected cells); NC, negative control (cells transfected with empty vectors).

growth and metastasis, and indicated poor survival of patients with retinoblastoma and gastric cancer $(15,16)$. Li et al (12) demonstrated that BANCR promoted cancer cell proliferation in malignant melanoma. Consistent with the aforementioned result, the current study revealed upregulated expression of BANCR in melanoma tissues compared with healthy adjacent tissues. Furthermore, overexpression of BANCR promoted proliferation of melanoma cells in vitro. The results obtained in the current study further demonstrated the oncogenic roles of BANCR in melanoma.

The oncogenic or tumor suppression roles of lncRNAs are achieved through the interactions with downstream tumor suppression or oncogenic pathways $(17,18)$. Previous studies have revealed that IncRNAs may interact with other non-coding RNAs, including microRNAs, to participate in cancer biology (17-19). However, studies on the interactions between different lncRNAs are rare. The present study revealed that LINC-PINT is downregulated in melanoma tissues compared with healthy adjacent tissues and may serve a role as tumor suppressor in this disease. Furthermore, the present study suggested that LINC-PINT may exert its effects in melanoma by serving as an upstream inhibitor of BANCR. BANCR has previously been revealed to activate the mitogen-activated protein kinase (MAPK) signaling pathway to promote the development of melanoma (12). A previous study 
A
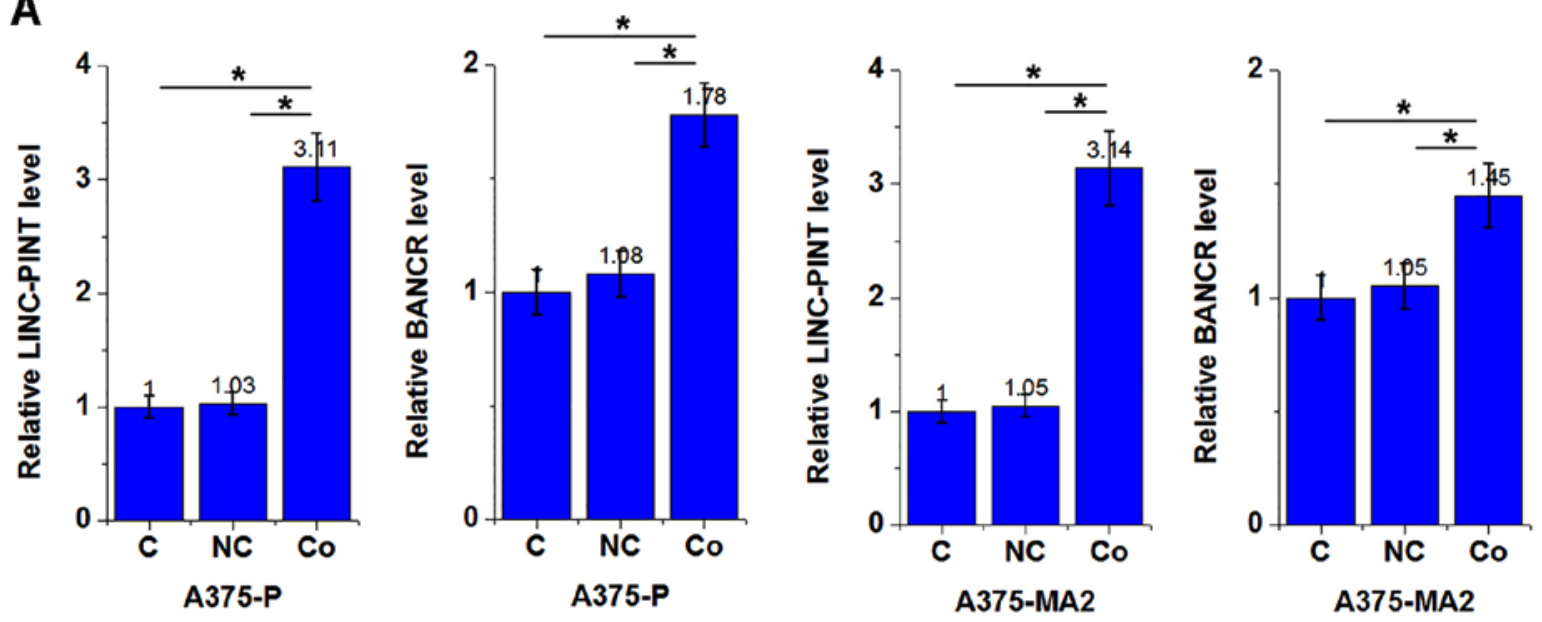

B

A375-P
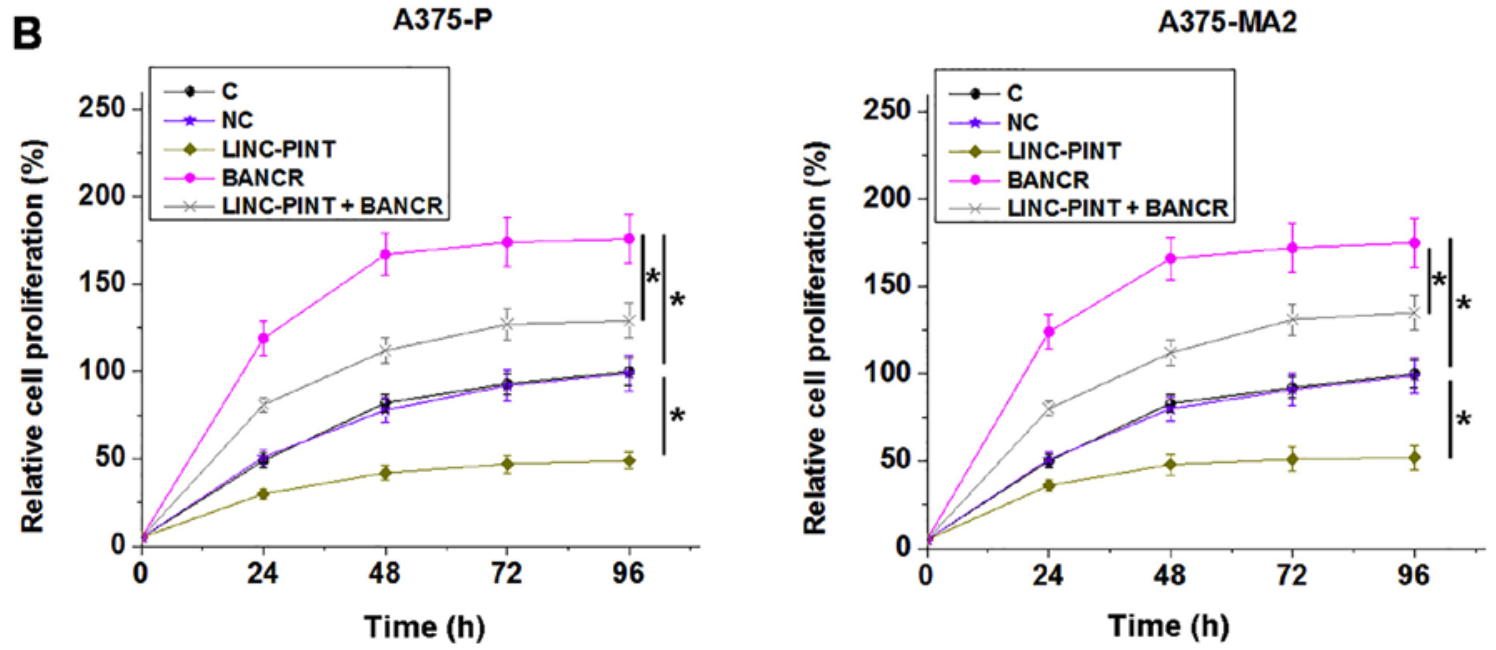

Figure 5. LINC-PINT overexpression inhibited melanoma cell proliferation through BANCR. (A) LINC-PINT and BANCR expression levels were significant increased following co-transfection with LINC-PINT and BANCR expression vectors. (B) Cell proliferation data revealed that LINC-PINT overexpression inhibited melanoma cell proliferation. BANCR overexpression exhibited an opposite effect and attenuated the effects of LINC-PINT overexpression. "P<0.05, as indicated. LINC-PINT, long intergenic non-protein coding RNA p53 induced transcript; BANCR, BRAF-activated non-protein coding RNA; C, control (untransfected cells); NC, negative control (cells transfected with empty vectors); Co, cells co-transfected with LINC-PINT and BANCR.

demonstrated that LINC-PINT interacts with MAPK in acute myocardial infarction (20). Additionally, BANCR has been reported to interact with MAPK (13). Therefore, LINC-PINT may interact the BANCR/MAPK signaling pathway to inhibit melanoma cell proliferation, and MAPK may mediate the interaction between LINC-PINT and BANCR. However, the current study did not investigate the role of MAPK. Future studies are required to elucidate the role of MPAK in the interaction between LINC-PINT and BANCR. The results obtained in the current study enriched the understanding of the molecular mechanisms in melanoma.

Notably, LINC-PINT overexpression failed to significantly affect the migration and invasion of melanoma cells (data not shown). Therefore, LINC-PINT may specifically inhibit the proliferation, but not other behaviors, of melanoma cells.

In conclusion, LINC-PINT is downregulated in melanoma, and LINC-PINT overexpression may inhibit melanoma cell proliferation by downregulating BANCR. The current study suggested that LINC-PINT may serve as a potential therapeutic target for melanoma.

\section{Acknowledgements}

Not applicable.

\section{Funding}

No funding was received.

\section{Availability of data and materials}

The datasets used and/or analyzed during the current study are available from the corresponding author on reasonable request.

\section{Authors' contributions}

ML designed the study. QH, QD and DZ performed all the experiments, analyzed the data and were major contributors in writing the manuscript. All authors read and approved the final manuscript. 


\section{Ethics approval and consent to participate}

Ethical approval was obtained from the Ethics Committee of Chongqing Traditional Chinese Medicine Hospital. All the patients provided written informed consent for participation in this study.

\section{Patient consent for publication}

Not applicable.

\section{Competing interests}

The authors declare that they have no competing interests.

\section{References}

1. MacKie RM, Hauschild A and Eggermont AM: Epidemiology of invasive cutaneous melanoma. Ann Oncol 20 (Suppl 6): vil-vi7, 2009.

2. Lund VJ, Chisholm EJ, Howard DJ and Wei WI: Sinonasal malignant melanoma: An analysis of 115 cases assessing outcomes of surgery, postoperative radiotherapy and endoscopic resection. Rhinology 50: 203-210, 2012.

3. Hodi FS, O'Day SJ, McDermott DF, Weber RW, Sosman JA Haanen JB, Gonzalez R, Robert C, Schadendorf D, Hassel JC, et al: Improved survival with ipilimumab in patients with metastatic melanoma. N Engl J Med 363: 711-723, 2010.

4. Schadendorf D, Hodi FS, Robert C, Weber JS, Margolin K, Hamid O, Patt D, Chen TT, Berman DM and Wolchok JD: Pooled analysis of long-term survival data from phase II and phase III trials of ipilimumab in unresectable or metastatic melanoma. J Clin Oncol 33: 1889-1894, 2015.

5. Takata M, Murata H and Saida T: Molecular pathogenesis of malignant melanoma: A different perspective from the studies of melanocytic nevus and acral melanoma. Pigment Cell Melanoma Res 23: 64-71, 2010.

6. Fatica A and Bozzoni I: Long non-coding RNAs: New players in cell differentiation and development. Nat Rev Genet 15: 7-21, 2014.

7. Mercer TR, Dinger ME and Mattick JS: Long non-coding RNAs: Insights into functions. Nat Rev Genet 10: 155-159, 2009.

8. Li J, Xuan Z and Liu C: Long non-coding RNAs and complex human diseases. Int J Mol Sci 14: 18790-18808, 2013.
9. Qi P and Du X: The long non-coding RNAs, a new cancer diagnostic and therapeutic gold mine. Mod Pathol 26: 155-165, 2013.

10. Marín-Béjar O, Mas AM, González J, Martinez D, Athie A, Morales X, Galduroz M, Raimondi I, Grossi E, Guo S, et al: The human lncRNA LINC-PINT inhibits tumor cell invasion through a highly conserved sequence element. Genome Biol 18: $202,2017$.

11. Garitano-Trojaola A, José-Enériz ES, Ezponda T, Unfried JP, Carrasco-León A, Razquin N, Barriocanal M, Vilas-Zornoza A, Sangro B, Segura V, et al: Deregulation of linc-PINT in acute lymphoblastic leukemia is implicated in abnormal proliferation of leukemic cells. Oncotarget 9: 12842-12852, 2018.

12. Li R, Zhang L, Jia L, Duan Y, Li Y, Bao L and Sha N: Long non-coding RNA BANCR promotes proliferation in malignant melanoma by regulating MAPK pathway activation. PLoS One 9: e100893, 2014.

13. Gershenwald JE and Scolyer RA: Melanoma staging: American joint committee on cancer (AJCC) 8th edition and beyond. Ann Surg Oncol 25: 2105-2110, 2018.

14. Livak KJ and Schmittgen TD: Analysis of relative gene expression data using real-time quantitative PCR and the 2(-Delta Delta C(T)) method. Methods 25: 402-408, 2001.

15. Su S, Gao J, Wang T, Wang J, Li H and Wang Z: Long non-coding RNA BANCR regulates growth and metastasis and is associated with poor prognosis in retinoblastoma. Tumour Biol 36: 7205-7211, 2015.

16. Li L, Zhang L, Zhang Y and Zhou F: Increased expression of LncRNA BANCR is associated with clinical progression and poor prognosis in gastric cancer. Biomed Pharmacother 72: 109-112, 2015.

17. Spizzo R, Almeida MI, Colombatti A and Calin GA: Long non-coding RNAs and cancer: A new frontier of translational research? Oncogene 31: 4577-4587, 2012.

18. Gutschner T and Diederichs S: The hallmarks of cancer: A long non-coding RNA point of view. RNA Biol 9: 703-719, 2012.

19. Braconi C, Kogure T, Valeri N, Huang N, Nuovo G, Costinean S, Negrini M, Miotto E, Croce CM and Patel T: microRNA-29 can regulate expression of the long non-coding RNA gene MEG3 in hepatocellular cancer. Oncogene 30: 4750-4756, 2011.

20. Zhu J, Gu H, Lv X, Yuan C, Ni P and Liu F: LINC-PINT activates the mitogen-activated protein kinase pathway to promote acute myocardial infarction by regulating miR-208a-3p. Circ J 82: 2783-2792, 2018

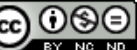

This work is licensed under a Creative Commons Attribution-NonCommercial-NoDerivatives 4.0 International (CC BY-NC-ND 4.0) License. 